WellBeing International

WBI Studies Repository

$9-2001$

\title{
The Effect of Response Contingencies on Scale Model Task Performance by Chimpanzees (Pan troglodytes)
}

Valerie A. Kuhlmeier

Yale University

Sarah T. Boysen

The Ohio State University

Follow this and additional works at: https://www.wellbeingintlstudiesrepository.org/acwp_asie

Part of the Animal Studies Commons, Comparative Psychology Commons, and the Other Animal Sciences Commons

\section{Recommended Citation}

Kuhlmeier, V. A., \& Boysen, S. T. (2001). The effect of response contingencies on scale model task performance by chimpanzees (Pan troglodytes). Journal of comparative psychology, 115(3), 300.

This material is brought to you for free and open access by WellBeing International. It has been accepted for inclusion by an authorized administrator of the WBI Studies Repository. For more information, please contact wbisr-info@wellbeingintl.org.

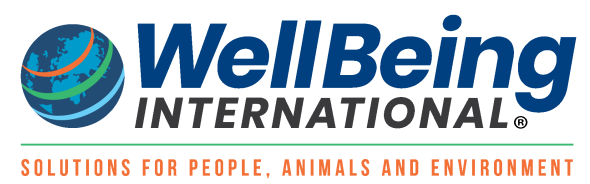




\title{
The Effect of Response Contingencies on Scale Model Task Performance by Chimpanzees (Pan troglodytes)
}

\author{
Valerie A. Kuhlmeier ${ }^{1}$ and Sarah T. Boysen ${ }^{2,3,4}$ \\ ${ }^{1}$ Yale University \\ ${ }^{2}$ Ohio State University \\ ${ }^{3}$ Emory University \\ ${ }^{4}$ Bioqual, Inc.
}

\begin{abstract}
$\underline{\text { ABSTRACT }}$
The effects of modified procedures on chimpanzees' (Pan troglodytes) performance in a scale model comprehension task were examined. Seven chimpanzees that previously participated in a task in which they searched an enclosure for a hidden item after watching an experimenter hide a miniature item in the analogous location in a scale model were retested under procedures incorporating response costs. In Experiment 1, chimpanzees were trained under procedures that rewarded only item retrievals occurring on the 1st search attempt. During test trials, 6 chimpanzees performed above chance, including 4 that were previously unsuccessful under the original procedures (V. A. Kuhlmeier, S. T. Boysen, \& K. L. Mukobi, 1999). Experiment 2 compared performance under the new and original procedures. Results indicated that for some chimpanzees, performance depended on procedures that decreased the use of competing search strategies and encouraged strategies based on information from the scale model.
\end{abstract}

Humans have devised various means of making it easier to navigate within the environment. For example, we create symbols to aid our memory of our surroundings and convey spatial information to others. Maps and scale models, for instance, portray landmarks and their spatial relationships to each other that are found in real space. Consequently, to understand and effectively use a physical representation of space, one has to recognize its correspondence with the real world. How and when we come to understand this correspondence has been the focus of much research in developmental psychology (e.g., Blades \& Cooke, 1994; DeLoache, 1987, 1991; Liben \& Downs, 1989; Huttenlocher, Newcombe, \& Vasilyeva, 1999).

DeLoache has approached this question in her innovative research program exploring children's ability to understand the representational nature of a scale model (e.g., DeLoache, 1987; DeLoache, Kolstad, \& Anderson, 1991; Marzolf, DeLoache, \& Kolstad, 1995). She and her colleagues have found that after witnessing a miniature item being hidden in a scale model of a room, 3-year-olds can locate a full-size item hidden in the analogous location in the real room. However, slightly younger children, 2.5-year-olds, 
have difficulty with the task (e.g., DeLoache, 1987). Their difficulty with the task implies the lack of representational insight, or knowledge that the model and room are related as a symbol and referent (DeLoache, 1995a, 1995b). DeLoache and her colleagues have suggested that many factors can contribute to the development of this understanding, including the perceptual similarity between the model and its referent (DeLoache et al., 1991), experience with other symbol systems (Marzolf \& DeLoache, 1994; Troseth \& DeLoache, 1999), instruction on the nature of the model-referent relationship (DeLoache, Mendoza, \& Anderson, 1999), and the ability to form a dual representation or represent the model as an object unto itself as well as a symbol for something else (DeLoache, 1987, 1991).

Until recently, it was not known whether a nonhuman species could understand a physical representation of space such as a scale model and use it as a source of information regarding the environment. Kuhlmeier, Boysen, and Mukobi (1999) found that chimpanzees were able to solve a scale model task that was similar to DeLoache's (1987) procedure. After watching an experimenter hide a miniature bottle of juice within a scale model of an outdoor enclosure, three chimpanzees readily found the real juice bottle that was hidden in the analogous location in the actual enclosure, going to the correct site and retrieving the bottle immediately upon entering the enclosure. These chimpanzees performed similarly to the 3-year-old children in DeLoache's task and demonstrated the representational insight that the model and enclosure were related. However, the performance of the other four chimpanzees tested was poor or, at best, varied. Two of these four subjects performed at chance level across the two experiments. Of the other two subjects, one chimpanzee performed above chance on only one of the experiments, and the other chimpanzee approached statistical significance during both experiments. These four subjects did not appear to be relying on the model-to-referent mapping strategy observed with the other three chimpanzees and with most 3-year-old children tested by DeLoache. Instead, they often relied on a search strategy that consisted of searching the hiding site in the front left comer of the enclosure and continuing to search each site successively as they circled the room clockwise. The strategy survived the two experiments detailed in Kuhlmeier et al. (1999) as well as conditions under which the chimpanzees were tested immediately before the present study (Kuhlrneier, 2000). This strategy ultimately resulted in locating the food reward, but it remained unclear whether these subjects recognized the representational nature of the scale model.

The search strategy that was observed with these chimpanzees has not been reported in studies by DeLoache and her colleagues. The most common error pattern reported for young children has been revisiting the site that contained the hidden toy on the previous trial (e.g., DeLoache, 1991). However, there is some preliminary evidence that young children, including 3-year-olds who otherwise show strong performance on the task, may demonstrate a search pattern similar to that of the chimpanzees when allowed to search the room uninterrupted until finding the hidden toy ( $T$. Solomon, personal communication, December 13, 2000). The present study tests whether the search strategy observed for the previously unsuccessful chimpanzees was due to a general inability of these animals to understand the scale model or whether it indicated the use of an alternate strategy that, though not demonstrating an underlying capacity for model comprehension, still led to the retrieval of the food reward. Results favoring the former explanation would suggest that chimpanzees do not achieve representation insight with scale models as readily as young children and have strong individual differences in performance. Results favoring the latter, however, would indicate that the use of alternate search strategies may be an additional factor, similar to those that DeLoache and colleagues have detailed, that can affect scale model task performance.

The present experiments used a procedure that made the search strategy ineffective for the chimpanzees, and only a strategy based on mapping the model-room relation would result in reward. The new procedure modified the standard task parameters such that attainment of the juice reward was now 
contingent on locating an empty juice bottle hidden in the enclosure on the chimpanzee's first search attempt. Thus, choosing an incorrect hiding site resulted in no reward, and consequently, the juice reward was not immediately available on all trials because a cost was imposed on incorrect choices. If the previously unsuccessful chimpanzees were, in fact, incapable of achieving representational insight with the model, this new procedure would not result in improved performance, as the subjects would never develop a mapping strategy. Conversely, if these chimpanzees did have the cognitive capacity for scale model comprehension, the use of the mapping strategy would be favored over the search strategy, and performance would improve and generalize to other models. During Experiment 1, the chimpanzees participated in training trials using the new contingency procedures and were subsequently tested for generalization with a new set of hiding sites. During Experiment 2, performance on the contingency task was compared with performance under the original, standard scale model procedures.

\section{Experiment 1}

\section{Method}

Subjects. Seven chimpanzees (Pan troglodytes) that had participated in previous experiments using the scale model task (Kuhlmeier et al., 1999) served as subjects in the current study. The group consisted of 3 females that were previously successful on the scale model task (Abby, age 26 years; Sarah, age 40 years; and Sheba, age 18 years) and 4 males that were previously unsuccessful on the scale model task (Bobby, age 13 years; Darrell, age 19 years; Digger, age 11 years; and Kermit, age 19 years). All but 2 of the chimpanzees (Abby and Digger) also had previous experience on a range of cognitive tasks (e.g., Boysen, Berntson, \& Mukobi, 2001; Boysen, Mukobi, \& Berntson, 1999; Thompson, Oden, \& Boysen, 1997).

Materials. Similar to the procedure reported by Kuhlmeier et al. (1999), all testing occurred in an outdoor exercise enclosure $(3.25 \mathrm{~m} \times 5.48 \mathrm{~m})$ that was made visually inaccessible from adjacent enclosures by hanging canvas tarps on the inside of the wire mesh walls that separated various play areas in the entire enclosure. During training trials in Experiment 1, the hiding sites in the enclosure consisted of a black rubber tire, a large blue plastic barrel, a square red bin, and a green plastic children's pool. For subsequent testing, the hiding sites included a light blue plastic chair; a yellow sandbox; a dark red trash can lid; and a white, rectangular 12-gal storage bin. For both training and test trials, a 1:7 scale model of the outdoor area containing miniature versions of the hiding sites was presented to individual chimpanzees. The model toys were constructed of clay, plastic, or cardboard and were painted to match the full-size objects. A full-size, empty, plastic juice bottle was hidden in the outdoor enclosure in a different location on each trial, and a miniature version of the plastic bottle was used for hiding in the scale model.

Procedures. Each chimpanzee first completed 32 contingency training trials. During each training trial, the experimenter stood on a ladder adjacent to a metal mesh transfer chute that connected several outdoor cages. The scale model was positioned on the top of the ladder. An individual chimpanzee subject watched from the overhead chute as the experimenter placed a miniature version of a plastic juice bottle in one of the four possible hiding sites in the scale model. Next, the chimpanzee was given access to the real outdoor enclosure, where an empty, full-size plastic juice bottle had been hidden in the analogous location (each hiding site was used for eight trials, with trial order randomized). If the chimpanzee retrieved the empty bottle on its first attempt, it was encouraged (through pointing, gesturing, and vocal requests) to trade the empty bottle for a full bottle of juice, which it was permitted to drink (contingency training procedure). ${ }^{1}$ However, if the chimpanzee did not locate the correct hiding site on its first attempt, it was encouraged to return to the transfer chute, and the trial was repeated. Incorrect trials were 
immediately followed by one, or if necessary, two retrials, although the latter represented only $12.5 \%$ of trials. Responses on retrials, however, were not included in subsequent data analyses.

After the contingency training trials were completed, each chimpanzee completed 12 test trials using the contingency procedures. The number of trials was limited to 12 to minimize training effects. For these trials, a second novel set of hiding sites and miniatures was used to test for generalization. Each of the four new sites was used for hiding three times, with trial order randomized for each chimpanzee. The procedures used during test trials were similar to the contingency training, with the exception that no retrials were permitted.

Statistical analyses. For this and all subsequent experiments, only retrievals that occurred on the first search attempt were considered correct responses (CRs). All trials were videotaped using a Quasar Palmcorder (VM565; Quasar, Torrance, CA) video recorder positioned on a tripod in front of the enclosure. The chimpanzees' responses were also recorded on data sheets by a second experimenter who was naive to the hiding site in the model. For purposes of generating interobserver reliability data, a third experimenter, who was also naive to the hiding sites, viewed all videotapes and recorded the chimpanzees' responses. These data were then compared with data collected by the second experimenter during all testing; Cohen's kappa statistic $(K)$ was used as a measure of reliability between observers. $^{2}$

Because of the modest sample size and efforts to avoid violation of normality assumptions, nonparametric tests were used for all analyses with alpha levels set at .05. To analyze individual responses, we adopted criteria based on the binomial distribution and a chance level equal to .25. At the group level, the sign test was used to determine statistical significance, whereas Mann-Whitney $U$ tests were used for testing differences between independent groups, and Wilcoxon tests examined differences between dependent groups.

\section{Results and Discussion}

After 32 training trials, each chimpanzee was performing above chance with the contingency procedure (each chimpanzee: $C R \geq 15$; binomial test, $p<.01$ ). That is, after watching the experimenter hide a miniature bottle in the scale model, chimpanzees were able to find the empty bottle hidden in the analogous location in the full-size enclosure on their first search attempt. Performance also improved over trials; the group median number of correct responses rose from 4 for the first eight trials $(50 \% \mathrm{CR})$ to 6 for the last block of eight trials (75\%; see Table 1). A Mann-Whitney $U$ test revealed no differences during training in number of correct responses between the chimpanzees that had been previously unsuccessful on the scale model task and those that were previously successful. Although one might expect that the previously successful chimpanzees would perform better in the early blocks than would the previously unsuccessful group, this was not the case. The change in procedure (i.e., having to bring the empty bottle to the experimenter) was new to these chimpanzees as well and might have led to initial confusion.

Differences between the previously successful and previously unsuccessful groups did emerge in the analysis of error patterns, however. Across all 32 training trials, the previously unsuccessful chimpanzees (Bobby, Darrell, Digger, and Kermit) performed the patterned searches on incorrect trials more frequently than the three previously successful chimpanzees (Abby, Sarah, and Sheba; Mann-Whitney $U=.05, p=$ .05). Comparing the first half of training trials with the second half indicated that this difference in search strategy frequency was only statistically significant in the first half of the trials (Mann-Whitney $U=.50, p=$ .05). During the second half of trials, both groups of chimpanzees were using the search strategy equally and infrequently. Thus, after 32 training trials, previously unsuccessful chimpanzees' behavior was similar 
to that of the previously successful chimpanzees, with both groups' performance indicating the use of the scale model as a source of information.

Table 1. Number of Correct Responses for Each Chimpanzee per Block of Eight Training Trials, Experiment 1

\begin{tabular}{|lcccc|}
\hline & \multicolumn{3}{c|}{ Trials } \\
\cline { 2 - 5 } Subject & $\mathbf{1 - 8}$ & $\mathbf{9 - 1 6}$ & $\mathbf{1 7 - 2 4}$ & $\mathbf{2 5 - 3 2}$ \\
\hline Abby & 4 & 4 & 5 & 6 \\
Sarah & 4 & 4 & 4 & 5 \\
Sheba & 3 & 3 & 5 & 5 \\
Bobby & 3 & 4 & 6 & 5 \\
Darrell & 5 & 4 & 5 & 7 \\
Digger & 3 & 4 & 6 & 5 \\
Kermit & 4 & 5 & 6 \\
\hline
\end{tabular}

Note. Five successful trials of eight, with chance level equal to .25 , constituted a reliable, above-chance performance (binomial test, $p<.05$ ).

For analyses across all training trials, 13 correct responses of 32 trials corresponded to $p<.05$ (binomial test). All chimpanzees had $\geq 15$ correct responses during the training trials.

Table 2. Number of Correct Responses for Each Chimpanzee During 12 Test Trials, Experiment 1

\begin{tabular}{|lc|}
\hline Subject & No. of correct responses \\
\hline Abby & 2 \\
Sarah & $6^{\star}$ \\
Sheba & $6^{\star}$ \\
Bobby & $10^{\star}$ \\
Darrell & $9^{\star}$ \\
Digger & $6^{\star}$ \\
Kermit & $9^{\star}$ \\
\hline
\end{tabular}

Note. Six successful trials out of 12 , with chance level equal to .25 , constituted a reliable, above-chance performance (binomial test, $p<.05$ ).

${ }^{*} p<.05$.

During the subsequent 12 test trials, which used a model and enclosure with four novel sites, all but one chimpanzee (Abby) continued to locate the empty, hidden bottle at levels above chance (for each: CR $\geq 6$ of 12 trials, $p<.05$; Table 2). Overall, the chimpanzees performed above chance at the group level (6 of 7 chimpanzees above chance, $p<.05)$. Furthermore, performance was constant across the trials, with no difference in number of correct responses between the first and second halves of trials. Similar to performance on the contingency training trials, there was no difference in performance between the previously successful and previously unsuccessful chimpanzees. The frequency of search strategy use was low and not significantly different between these two groups (median for previously unsuccessful $=1$, median for previously successful $=1$ ).

Thus, under the new contingency procedure, not only did 2 of 3 chimpanzees that were previously successful on the scale model task (Kuhlmeier et al., 1999) replicate their previous performance, but previously unsuccessful chimpanzees performed equally as well as they did. These chimpanzees 
switched from a strategy based on searching each site successively to a mapping strategy in which information from the model was used. Consequently, their poor performance in the earlier study (Kuhlmeier et al., 1999) was not due to a general inability to recognize the model's relation to the room but was most likely due to the use of an alternate search strategy that used information from the model.

\section{Experiment 2}

Given the chimpanzees' successful performance when tested under the contingency procedure, Experiment 2 was designed to make direct comparisons of the chimpanzees' performance under the contingency and standard procedures (during which a full juice bottle was hidden). All chimpanzees were tested under both procedures to evaluate whether the previously unsuccessful chimpanzees (see Kuhlmeier et al., 1999) would revert back to the search strategies observed under the standard scale model conditions or whether the mapping behavior demonstrated under the contingency conditions would show transfer.

\section{Method}

During Experiment 2, four novel hiding sites were used, including a blue plastic trash can lid; a brown plastic trash can; a pink plastic children's slide; and a large, wood cable spool. The scale model contained miniature versions of these objects. Each chimpanzee completed a set of eight trials under the standard procedure, in which a full juice bottle was hidden within the enclosure, and a set of eight trials under the contingency procedure, in which an empty bottle was hidden. Each site served as the hiding location for the empty or full juice bottle on 4 of the 16 total trials, with trial order randomized for each chimpanzee. Three chimpanzees completed eight contingency trials first, followed by eight standard task trials; the remaining 4 chimpanzees received the trials in the opposite order. ${ }^{3}$

\section{Results and Discussion}

Under both procedures, the chimpanzees performed significantly above chance at the group level (standard procedure: 5 of 7 chimpanzees performed above chance, $p<.05$; contingency procedure: 6 of 7 chimpanzees performed above chance, $p<.05$ ). At the individual level, 5 of 7 chimpanzees were successful with the task under the standard procedure (Bobby, Darrell, Kermit, Sarah, and Sheba: CR $\geq 5$ of 8 trials, $p<.05$ ), whereas 2 chimpanzees' (Abby and Digger) performance was not above chance level. Similarly, under the contingency procedure, all chimpanzees but Digger performed successfully on the task ( 6 of 7 chimpanzees: $\mathrm{CR} \geq 5$ of 8 trials, $p<.05$; Table 3 ). A Wilcoxon test comparing performance on both procedures yielded no significant difference (median percentage correct responses for both procedures $=62.5 \%$ ), and there was no significant effect of procedure order.

Analyses comparing the performance of the previously successful and previously unsuccessful chimpanzees indicated that there was no difference in performance under either procedure. Furthermore, the search strategy was used minimally by both of these groups. Across all 7 chimpanzees, only 17 of 43 (16.3\%) incorrect trials were due to using the search strategy, and there was no difference in the frequency of search strategy use between the groups or between the test procedures.

Two chimpanzees, however, demonstrated poor performance during these trials. Digger's poor performance was surprising, given his success during Experiment 1. During the contingency trials in Experiment 2, there were new behavioral indications that Digger had become frustrated, particularly in response to errors and the subsequent cost now associated with incorrect responses. If incorrect, Digger would often exhibit species-typical display behavior, including charging, piloerection, and pant-hooting, which are natural chimpanzee vocalizations produced under circumstances of high arousal. During such displays, Digger also hurled the hiding sites around the enclosure. These behaviors during testing were 
unique to Digger. Digger's species-typical display behavior suggested that he was experiencing great frustration associated with nonreward, and his behavioral responses likely interfered with success on the task (see, e.g., Amsel, 1958, 1990; Douglas, 1985). Digger, who had performed at 50\% correct throughout the contingency training and testing (Experiment 1), may have reacted with more frustration to the loss of reward on these and subsequent trials than the other chimpanzees. The determinants of the poor performance of Abby, however, are less clear. Abby's performance during the original scale model task (Kuhlmeier et al., 1999) and the contingency training in Experiment 1 of the present study suggested that she had the ability to use the model as a source of information regarding analogous hiding sites. Thus, the reason or reasons for Abby's deteriorated performance during the present experiments remain unclear.

Table 3. Number of Correct Responses for Each Chimpanzee During the Eight Standard Procedure Trials and Eight Contingency Procedure Trials of Experiment 2

\begin{tabular}{|lcc|}
\hline & \multicolumn{3}{c|}{ No. of correct responses } \\
\cline { 2 - 3 } Subject & Standard & Contingency \\
\hline Abby & 3 & $5^{\star}$ \\
Sarah & $5^{*}$ & $6^{\star}$ \\
Sheba & $6^{*}$ & $5^{\star}$ \\
Bobby & $5^{\star}$ & $5^{\star}$ \\
Darrell & $7^{*}$ & $7^{\star}$ \\
Digger & 0 & 3 \\
Kermit & $5^{*}$ & $6^{\star}$ \\
\hline
\end{tabular}

Note. Five successful trials of eight, with chance level equal to .25 , constituted a reliable, above-chance performance (binomial test, $p<.05$ ).

${ }^{*} p<.05$.

Overall, 5 of the 7 chimpanzees were able to solve the scale model task under conditions in which the full juice bottle was not present (contingency procedure) and also under conditions during which 3 of these chimpanzees had previously shown consistently poor performance (standard procedure; see Kuhlmeier et al., 1999). The results thus replicate the findings of Experiment 1 and extend them to a different task procedure. It is important to note that the chimpanzees that previously relied on the alternate search strategy did not revert to this strategy during the eight trials using the standard task procedure, even though use of this strategy would have resulted in obtaining the juice reward. Instead, these chimpanzees continued to use the newly acquired mapping strategy. It is possible that the mapping strategy was maintained because of its efficiency. Measurement of latency for bottle retrieval for a sample of 20 standard procedure trials found that a response due to mapping averaged $4 \mathrm{~s}$ to complete, whereas searching strategies took an average of $6 \mathrm{~s}$ (if completed at the second site) or $12 \mathrm{~s}$ (if completed at the fourth site). Thus, search strategies took an average of 2 to $8 \mathrm{~s}$ more for the chimpanzees to retrieve the bottle. This difference may have been detectable to the chimpanzees; however, it is uncertain whether this difference in latency to reward is large enough to affect performance.

An alternate possibility is that not enough trials were completed using the standard procedure to demonstrate a shift back to the search strategy. Indeed, for 3 of the 4 previously unsuccessful chimpanzees, the strategy was not observed in the current standard procedure trials until the second half of the trials. Thus, although the mapping strategy did transfer to the standard procedure for the eight trials that were run, the search strategy began to appear toward the end of these trials, indicating that after more trials, this alternate strategy might have been used. 


\section{General Discussion}

The present experiments suggest that for chimpanzees that previously had low performance on the scale model task, manipulation of the task demands led to performance that demonstrated an ability to use the model as a source of information. In previous experiments using the standard task (Kuhlmeier et al., 1999), during which a reward was present and obtainable on every trial, some chimpanzees failed to perform in a manner that demonstrated scale model comprehension (i.e., locating the hidden object during a search of the first selected site). In contrast, during training under the contingency conditions of the present experiments, all 7 chimpanzees solved the task at levels above chance. Performance rose quickly during training, with all 4 previously unsuccessful chimpanzees demonstrating at least $50 \%$ correct retrievals after only 16 training trials. Furthermore, these chimpanzees performed similarly to the previously successful chimpanzees during the training trials. On subsequent novel test trials (Experiment 1), all but 1 of the 7 chimpanzees were able to find the empty bottle immediately after entering the enclosure. During subsequent testing under the standard and contingency conditions in Experiment 2, 5 of 7 chimpanzee subjects continued to show strong performance on the scale model task. Thus, under the procedures of the contingency manipulation, all the chimpanzees readily demonstrated model-toroom mapping behavior, suggesting a capacity, previously undetected in some chimpanzees, to recognize the correspondence between the model and the corresponding full-size enclosure.

Thus, the present experiments have offered further support that chimpanzees can achieve representational insight with a scale model, similar to that proposed for 3-year-old children. Now, all 7 chimpanzees tested demonstrated scale model comprehension. Additionally, the experiments have indicated a factor that may affect task performance for chimpanzees, namely the use of an alternate strategy that does not require mapping the model-room relationship. Results from both current experiments demonstrate that understanding the representational nature of the model was not beyond the cognitive grasp of 4 chimpanzees that were previously unsuccessful in the Kuhlmeier et al. (1999) study. Instead, their reliance on an alternative search strategy made the model irrelevant to these chimpanzees and, thus, experimental detection of representational insight impossible. In the present study, however, they demonstrated an understanding of the relevance of the scale model.

Given their rapid improvement in performance during training and subsequent testing, the question remains whether the previously unsuccessful chimpanzees detected the model-room relationship early in testing (e.g., during Kuhlmeier et al., 1999) and chose instead to rely on an alternate search strategy or whether representational insight was only achieved through the contingency training in the present study. Several factors suggest that the former is true and that previously observed differences in performance were due to alternate strategies, not underlying differences in representational insight ability. For example, as detailed in Kuhlmeier et al. (1999), 2 of the 4 chimpanzees that frequently used the search strategy demonstrated some indications of scale model comprehension during this earlier study. Specifically, 1 of these chimpanzees, Darrell, performed well during the first experiment but then relied frequently on the search strategy during the second experiment. The other chimpanzee, Kermit, performed at 50\% correct responses during the second experiment, only one trial short of the criterion for statistical significance. Thus, it is possible that these chimpanzees did recognize the representational nature of the model.

Another factor supporting early insight is the strong performance of the 4 previously unsuccessful chimpanzees during the training trials in the present study. Two of these 4 chimpanzees performed above chance after only eight trials, and all 4 were performing at or above $50 \%$ by only the 16th training trial. Additionally, the performance of these 4 chimpanzees did not differ from that of the 3 previously successful chimpanzees. Consequently, the increasing performance during these trials might better be described as improved understanding of the task requirements rather than learning of the model-room 
relationship. Additional support for an early-insight explanation comes from evidence that the mapping strategy and the search strategy can coexist as retrieval alternatives. Although the frequency of use of the search strategy was low when the contingency procedure was in place, error analyses indicated that this strategy was occasionally attempted even though chimpanzees were demonstrating scale model comprehension on the whole. Preliminary evidence from studies with young children also indicates that 3year-olds, an age group repeatedly observed to understand scale models (e.g., DeLoache, 1987, 1991), will often demonstrate this pattern of search on incorrect trials (T. Solomon, personal communication, December 13, 2000). Thus, these three factors support the premise that previously unsuccessful chimpanzees in Kuhlmeier et al. (1999) did have an underlying ability to comprehend the scale model early on and that poor performance was due to the use of an alternate strategy.

However, there are two arguments that can be made against this explanation. First, the previously unsuccessful chimpanzees did not immediately revert back to the search strategy during the standard task procedure condition of Experiment 2. These results could imply that contrary to an early-insight explanation, the contingency experience led to representational insight, which influenced the use of a model-to-room mapping strategy even on trials in which it was not required to achieve the juice reward. However, it is important to note that when the search strategy did appear during the standard task procedure trials, it was during the second half of trials. This suggests that only eight test trials (a limit that was required to minimize the effects of training) may not have been enough trials to observe some of the chimpanzees returning to the search strategy. A second argument against the early-insight explanation questions why these chimpanzees chose the search strategy over the mapping strategy during the Kuhlmeier et al. (1999) study, given that they understood that the model, if attended to, would offer information that could lead to more efficient searching. One possibility is that although the mapping strategy is more efficient because it offers a slight gain in time to reward (2-8 s), it may be a more cognitively demanding strategy because, for example, of the need to retain the encoded model information on a given trial and possible proactive interference between trials. Thus, given the cognitive demands inherent to the mapping strategy, the search strategy might have provided an easier manner to find the juice reward and consequently would have been the preferred strategy for some chimpanzees.

It is important to note that the previously unsuccessful chimpanzees in Kuhlmeier et al. (1999) were all male and that only the female chimpanzees demonstrated a stable model-to-room mapping strategy. Although conclusions regarding sex differences that are drawn from only 7 chimpanzees are necessarily limited, it is telling that of the many cognitive tasks in which these animals have participated (e.g., Boysen, Berntson, Hannan, \& Cacioppo, 1996; Thompson et al., 1997), the Kuhlmeier et al. (1999) study was the first to report a difference such as this among the chimpanzees. Scale model research with young children has made some suggestion of sex differences in performance. Although DeLoache did not find sex differences in performance on the original scale model task (e.g., 1987, 1991), girls have performed better than boys in two subsequent manipulations of her original task. Marzolf and DeLoache (1994), for instance, found a sex difference with a task designed to explore the transfer of performance from the task that is usually solved by young children (the scale model task) to a task with which they had difficulty (using a map). Among the children tested, young girls (3 years old) showed greater improvement in performance on the map task after experience with the scale model task than age-matched boys did. Furthermore, using a task similar to DeLoache's standard task (e.g., 1987), Blades and Cooke (1994) found that girls ( 3 years old) performed better than boys of the same age.

One possible reason for this sex difference among children and chimpanzees may be due to underlying differences in how aspects of the model are attended to and used. A scale model and its referent are related in terms of the similarity of the objects within them (landmark or object correspondence) as well as in terms of the similarity in the spatial layout of these objects (geometric or relational correspondence). 
Thus, both objects and spatial layout provide cues to the overall relationship between the model and its referent. Studies with children (Marzolf, DeLoache, \& Kolstad, 1999) and chimpanzees (Kuhlmeier \& Boysen, in press) have demonstrated that although both children and chimpanzees recognize both of these types of correspondences, object cues are more salient and play a more powerful role in successful task performance (indeed, the hiding site itself is a landmark in the room). However, males and females may differ in their reliance on object and spatial cues in general. For example, in navigational tasks that do not use models or other physical representations of space, females of many species demonstrate a stronger reliance on object and landmark cues than on spatial and relational cues, whereas males demonstrate the opposite preference (e.g., Sandstrom, Kaufman, \& Heuttel, 1998; Williams, Barnett, \& Meek, 1990; Williams \& Meek, 1991). In tasks using scale models, there is also some suggestion that girls attend more to object cues, whereas boys attend more to spatial cues. For example, 3-year-old girls outperformed boys in the second of two experiments reported by Marzolf, DeLoache, and Kolstad (1999) in which a scale model and a full-size room had different furniture arrangements. That is, boys did not perform as well as girls did when spatial cues were eliminated and only object cues remained, suggesting that boys required the presence of spatial cues, whereas girls did not. Similarly, preliminary analyses from another study indicate that 3-year-old children rely primarily on object cues to solve the task; however, the effect is qualified by sex, with girls attending more to objects and with boys, in some conditions, attending more to spatial cues (T. Solomon, personal communication, December 13, 2000). Thus, in a task in which object cues play a prominent role, males may be contraprepared for successful performance. Consequently, the male chimpanzees' previous reliance on a strategy other than mapping may have been the result of the task being more difficult for them than for female chimpanzees. This cognitive demand, as well as the cognitive demands inherent to the task and described above, may have led to the favoring of an alternate search strategy that did not rely on the scale model.

In conclusion, the present experiments replicate and extend previous studies of chimpanzee scale model comprehension (Kuhlmeier et al., 1999), demonstrating that chimpanzees are capable of understanding a physical representation of space. Furthermore, they present another factor that can potentially impede the experimental detection of scale model comprehension, that is, the use of an alternate search strategy that does not necessarily lead to correct item retrieval on the first search attempt. There is some suggestion that young children might also demonstrate this type of search behavior ( $T$. Solomon, personal communication, December 13, 2000), yet perhaps not with the same frequency as chimpanzees. It will be informative to examine the performance of young children using task procedures similar to that of the contingency procedure to determine whether the requirement of first-search retrieval can improve performance.

\section{Notes}

${ }^{1}$ The chimpanzees had many years of experience trading objects in their enclosure for food rewards, and none had difficulty generalizing this behavior to the current task.

${ }^{2}$ In Experiments 1 and 2, interobserver reliability measures yielded $K \geq .98$, indicating strong agreement between observers for the chimpanzees' search choices. Thus, for conciseness in reporting results, we do not report the kappa values individually.

${ }^{3}$ The two groups were constructed such that the chimpanzees' age and sex were as evenly distributed as possible (Group 1: Darrell, Digger, and Sarah; Group 2: Abby, Bobby, Kermit, and Sheba). 


\section{References}

Amsel, A. (1958). The role of frustrative nonreward in noncontinuous reward situations. Psychological Bulletin, 55, 102-119.

Amsel, A. (1990). Arousal, suppression, and persistence: Frustration theory, attention and its disorders. Cognition and Emotion, 4, 239-269.

Blades, M., \& Cooke, Z. (1994). Young children's ability to understand a model as a spatial representation. Journal of Genetic Psychology, 155, 201-218.

Boysen, S. T., Berntson, G. G., Hannan, M. B., \& Cacioppo, J. T. (1996). Quantity-based interference and symbolic representations in chimpanzees (Pan troglodytes). Journal of Experimental Psychology: Animal Behavior Processes, 22, 76-86.

Boysen, S. T., Berntson, G. G., \& Mukobi, K. M. (2001). Size matters: Impact of item size and quantity on array choice by chimpanzees (Pan troglodytes). Journal of Comparative Psychology, 115, 106110.

Boysen, S. T., Mukobi, K. L., \& Berntson, G. G. (1999). Overcoming response-bias using symbolic representations of number by chimpanzees (Pan troglodytes). Animal Learning and Behavior, 27, 229-235.

DeLoache, J. S. (1987). Rapid change in the symbolic functioning of very young children. Science, 238, 1556-1557.

DeLoache, J. S. (1991). Symbolic functioning in very young children: Understanding of pictures and models. Child Development, 62, 736-752.

DeLoache, J. S. (1995a). Early symbolic understanding and use. In D. Medin (Ed.), The psychology of learning and motivation (Vol. 33, pp. 65-114). New York: Academic Press.

DeLoache, J. S. (1995b). Early understanding and use of symbols. Current Directions in Psychological Science, 4, 109-113.

DeLoache, J. S., Kolstad, D. V., \& Anderson, K. N. (1991). Physical similarity and young children's understanding of scale models. Child Development, 62, 111-126.

DeLoache, J. S. , Mendoza, O. A., \& Anderson, K. N. (1999). Multiple factors in early symbol use: Instructions, similarity, and age in understanding a symbol-referent relation. Cognitive Development, 14, 299-312.

Douglas, V. I. ( 1985). The response of ADD children to reinforcement: Theoretical and clinical implications. In L. M. Bloomingdale (Ed.), Attention deficit disorder: Identification, course and rationale (pp. 49-66). New York: Spectrum.

Huttenlocher, J., Newcombe, N., \& Vasilyeva, M. (1999). Spatial scaling in young children. Psychological Science, 10, 393-398.

Kuhlmeier, V. A. (2000). Use of a physical representation of space by chimpanzees (Pan troglodytes): Comparative perspectives on scale model comprehension. Unpublished doctoral dissertation, Ohio State University.

Kuhlmeier, V. A., \& Boysen, S. T. (in press). Chimpanzees (Pan troglodytes) recognize spatial and object correspondences between a scale model and its referent. Psychological Science.

Kuhlmeier, V. A., Boysen, S. T., \& Mukobi, K. L. (1999). Scale model comprehension by chimpanzees (Pan troglodytes). Journal of Comparative Psychology, 113, 396-402.

Liben, L., \& Downs, R. (1989). Understanding maps as symbols. In H. W. Reese (Ed.), Advances in child development and behavior (Vol. 22, pp. 145-201). New York: Academic Press.

Marzolf, D. P., \& DeLoache, J. S. (1994). Transfer in young children's understanding of spatial representations. Child Development, 65, 1-15.

Marzolf, D. P., DeLoache, J. S., \& Kolstad, D. V. (1999). The role of relational similarity in young children's use of a scale model. Developmental Science, 2, 296-305. 
Sandstrom, N. J., Kaufman, J., \& Huettel, S. A. (1998). Males and females use different distal cues in a virtual environment navigation task. Cognitive Brain Research, 6, 351-360.

Thompson, R. K. R., Oden, D. L., \& Boysen, S. T. (1997). Language-naïve chimpanzees (Pan troglodytes) judge relations-between-relations in an abstract matching task. Journal of Experimental Psychology: Animal Behavior Processes, 23, 31-43.

Troseth, G., \& DeLoache, J. S. (1999). The medium can obscure the message: Young children's understanding of video. Child Development, 69, 950-965.

Williams, C. L., Barnett, A. M., \& Meek, W. H. (1990). Organizational effects of early gonadal secretions on sexual differentiation in spatial memory. Behavioral Neuroscience, 104, 84-97.

Williams, C. L., \& Meek, W. H. (1991). The organizational effects of gonadal steroids on sexually dimorphic spatial ability. Psychoneuroendocrinology, 16, 155-176. 